\title{
In vitro Degradation of Antimicrobials during Use of Broth Microdilution Method Can Increase the Measured Minimal Inhibitory and Minimal Bactericidal Concentrations
}

\author{
Elodie A. Lallemand ${ }^{1 *}$, Marlène Z. Lacroix ${ }^{1}$, Pierre-Louis Toutain ${ }^{1}$, Séverine Boullier ${ }^{2}$, \\ Aude A. Ferran ${ }^{1}$ and Alain Bousquet-Melou ${ }^{1}$ \\ 'TOXALIM, Université de Toulouse, Institut National de la Recherche Agronomique, ENVT, INP-Purpan, UPS, Toulouse, \\ France, ${ }^{2}$ Université de Toulouse, ENVT, Toulouse, France
}

OPEN ACCESS

Edited by:

Octavio Luiz Franco, Universidade Católica de Brasilia,

Brazil

Reviewed by:

Patrícia Poeta,

University of Trás-os-Montes and Alto

Douro, Portugal

Chetan Sharma,

Guru Angad Dev Veterinary and Animal Sciences University, India

${ }^{*}$ Correspondence: Elodie A. Lallemand e.lallemand@envt.fr

Specialty section:

This article was submitted to Antimicrobials, Resistance and

Chemotherapy,

a section of the journal

Frontiers in Microbiology

Received: 28 October 2016 Accepted: 07 December 2016 Published: 21 December 2016

Citation:

Lallemand EA, Lacroix MZ Toutain P-L, Boullier S, Ferran AA and Bousquet-Melou A (2016) In vitro

Degradation of Antimicrobials during Use of Broth Microdilution Method Can Increase the Measured Minimal Inhibitory and Minimal Bactericidal

Concentrations.

Front. Microbiol. 7:2051.

doi: 10.3389/fmicb.2016.02051
The antibacterial activity of some antimicrobials may be under-estimated during in vitro microbiological susceptibility tests, due to their instability under such conditions. The in vitro degradation of seven widely used antimicrobials (amoxicillin, cephalexin monohydrate, cefotaxime sodium salt, ciprofloxacin, erythromycin hydrate, clarithromycin, and doxycycline hyclate) and its effect on MIC and MBC determinations was studied using the broth microdilution method, considered as the gold standard for MIC determinations. In vitro concentrations of antimicrobials, over a $24 \mathrm{~h}$ incubation period in the medium tested without bacteria, decreased from 33\% for ciprofloxacin to $69 \%$ for clarithromycin. For cephalexin, cefotaxime, clarithromycin, and doxycycline which were the most degraded drugs, MIC and MBC values for one strain of $E$. coli and one strain of $S$. aureus were compared using the standard method or after ad-hoc drug complementation aiming at maintaining constant drug concentration. Abiotic degradation during the standard method was associated with a significant increase of the MIC (2 antibiotics) and MBC (3 antibiotics). However, the observed discrepancy (less than one twofold dilution), even for the most degraded drug for which the concentration at $24 \mathrm{~h}$ was reduced by two thirds, suggests that this would only be clinically significant in special cases such as slow-growing bacteria.

Keywords: antimicrobial, stability, degradation, MIC, MBC

\section{INTRODUCTION}

The minimal inhibitory concentration (MIC) is the lowest concentration of an antimicrobial that inhibits visible growth of a bacterial culture under a defined set of experimental conditions (Clinical and Laboratory Standards Institute, 2012). One reference method for measuring MIC is broth microdilution under standardized in vitro conditions, using a microdilution tray with twofold drug-dilution steps, and subsequent visual evaluation of bacterial growth or non-growth after $16-20 \mathrm{~h}$ of culture at $35 \pm 2^{\circ} \mathrm{C}$ (Clinical and Laboratory Standards Institute, 2012). It is assumed that the in vitro concentration of the tested antimicrobial remains unchanged throughout the incubation period. However, the in vitro stability of antimicrobials is known to be affected by 
physical factors such as light, composition of the medium, $\mathrm{pH}$, and temperature (Paesen et al., 1994). Such instability may lead to underestimation of the antibacterial activity of the compound in Antimicrobial Susceptibility Tests (AST) (Marchbanks et al., 1987) and thus alter the clinical decision to increase the dose, add another antimicrobial agent, or even abandon therapy with the compound. It has already been shown that the storage conditions of prepared MIC trays can reduce the biological activity of antimicrobials, depending on the drug, storage duration and temperature (Barry et al., 1976; Nickolai et al., 1985; Hwang et al., 1986; White et al., 1991). Few studies have focused on the degradation of antimicrobials during the actual incubation period (Wick, 1964; Marchbanks et al., 1987) and we are not aware of any study of the subsequent effect on minimal bactericidal concentration (MBC) determinations.

The aim of this study was to see if the incubation conditions used during the microdilution test could lead to the substantial degradation of some antimicrobials and if such degradation could affect the AST results.

\section{MATERIALS AND METHODS}

\section{Antimicrobial Degradation Experiment}

The tested drugs were selected among antimicrobials frequently used in human and veterinary medicine, without regard to their reported stability status. The drugs and dilutions in the microplates were as follows: Cephalexin monohydrate 0.5$256 \mu \mathrm{g} / \mathrm{mL}$, amoxicillin $0.25-128 \mu \mathrm{g} / \mathrm{mL}$, cefotaxime sodium salt $0.125-64 \mu \mathrm{g} / \mathrm{mL}$, ciprofloxacin $0.031-16 \mu \mathrm{g} / \mathrm{mL}$, erythromycin hydrate and clarithromycin $0.016-8 \mu \mathrm{g} / \mathrm{mL}$ and doxycycline hyclate $0.008-4 \mu \mathrm{g} / \mathrm{mL}$. All drugs were tested at twofold serial dilutions. The antimicrobial agents were dissolved according to the specifications of the manufacturers (Sigma Aldrich, Saint Louis, MO for amoxicillin, cefotaxime sodium salt, ciprofloxacin, doxycyline hyclate, erythromycin hydrate and clarithromycin; ACS Dobfar, Tribiano, Italy for cephalexin monohydrate). Microdilution trays were prepared extemporaneously as previously described for MIC determination (Nickolai et al., 1985), except that $100 \mu \mathrm{L}$ of cation-adjusted, organism-free Mueller-Hinton broth (MHB) was added to prevent any drug degradation that might be caused by bacteria. The trays were placed in a dark incubator at $37^{\circ} \mathrm{C}$. At each sampling time (i.e., $0,3,6,10$, and $24 \mathrm{~h}$ ), $100 \mu \mathrm{L}$ were collected from 3 adjacent wells within less than $2 \mathrm{~min}$, diluted with $900 \mu \mathrm{L}$ of $\mathrm{H}_{2} \mathrm{O}$ and stored at $4^{\circ} \mathrm{C}$ for less than $4 \mathrm{~h}$ before analysis.

\section{Antimicrobials Quantitative Analysis}

All antimicrobials were assayed in triplicate by LC/MS with an Acquity UPLC coupled to a Xevo triple quadrupole mass spectrometer (Waters, Milford, MA, USA). Five microliters of diluted sample were injected into a Cortecs C18 column $\left(2.1^{*} 50 \mathrm{~mm} ; 1.6 \mu \mathrm{m}\right.$, Waters) and eluted with an $\mathrm{H}_{2} \mathrm{O}, 0.1 \%$ formic acid and acetonitrile gradient (except for cephalexin which was assayed with an $\mathrm{H}_{2} \mathrm{O}, 0.1 \%$ formic acid/acétonitrile isocratic elution). The column and autosampler temperatures were set at 40 and $10^{\circ} \mathrm{C}$ respectively. Samples were ionized in positive electrospray ionization mode (ESI+). The capillary voltage and source temperature were set at $2.5 \mathrm{kV}$ and $150^{\circ} \mathrm{C}$ respectively. The desolvation temperature and nitrogen flow rate were set at $650^{\circ} \mathrm{C}$ and $800 \mathrm{~L} / \mathrm{h}$ respectively. Argon was used as the collision gas at a flow rate of $0.12 \mathrm{~mL} / \mathrm{min}$. Quantification was by multiple reactions monitoring (MRM). The MRM transitions, cone voltage and collision energies used for the different antimicrobials are reported in Table 1. The method was validated in terms of linearity, inter-day and intraday repeatability, selectivity and sensitivity, for each molecule (ICH, 1994). The calibration curve was obtained by injecting 3 replicates of seven calibration standards prepared in $\mathrm{MHB}$ and ranging from 0.01 to $50 \mu \mathrm{g} / \mathrm{mL}$. Three different approaches were used to determine the linearity of the calibration curve: (1) calculation of the relative concentration residuals between the nominal concentration and the concentration obtained with the model (RCR\%), (2) visual inspection of the residual distribution, and (3) application of a lack of fit test to check the goodness-of-fit of the model (Almeida et al., 2002). The selectivity of the method was characterized by injecting 6 replicates of MHB blank samples and comparing the obtained signal with those obtained at the limit of quantification (LOQ). The LOQ of each antimicrobial was the lowest concentration on the calibration curve that could be quantified with a precision lower than $20 \%$ and within an accuracy range of $80-120 \%$. Calculations of intra-day and interday precisions and accuracies for each antimicrobial were based on three different days (except for cephalexin: 2 days) and with six replicates of QC samples at three concentration levels (low, middle, and high) covering the range of standard curve concentrations.

\section{Effect of Antimicrobial Degradation on AST}

The effects of antimicrobial degradation on AST were further investigated by using the standard microdilution technique (Clinical and Laboratory Standards Institute, 2012) with or without an ad-hoc compensatory addition of antimicrobials

TABLE 1 | Retention times, MRM transitions, and MS parameters of seven different antimicrobials.

\begin{tabular}{|c|c|c|c|c|c|}
\hline Antimicrobials & $\begin{array}{l}\text { Retention } \\
\text { time } \\
\text { (min) }\end{array}$ & $\begin{array}{c}\text { Ion } \\
\text { parent } \\
(\mathrm{m} / \mathrm{z})\end{array}$ & $\begin{array}{c}\text { Ion } \\
\text { daughter } \\
(\mathrm{m} / \mathrm{z})\end{array}$ & $\begin{array}{c}\text { Cone } \\
\text { voltage } \\
\text { (V) }\end{array}$ & $\begin{array}{c}\text { Collision } \\
\text { energy } \\
\text { (eV) }\end{array}$ \\
\hline \multirow[t]{2}{*}{ Amoxicillin } & 0.81 & 366.0 & 349.1 & 14 & 8 \\
\hline & & & 113.9 & & 22 \\
\hline \multirow[t]{2}{*}{ Cefotaxime } & 1.49 & 456.0 & 396.1 & 18 & 12 \\
\hline & & & 166.9 & & 20 \\
\hline \multirow[t]{2}{*}{ Ciprofloxacin } & 1.49 & 332.1 & 288.1 & 32 & 18 \\
\hline & & & 245.1 & & 24 \\
\hline \multirow[t]{2}{*}{ Doxycycline } & 2.27 & 445.1 & 428.2 & 24 & 18 \\
\hline & & & 154.0 & & 30 \\
\hline \multirow[t]{2}{*}{ Erythromycin } & 2.94 & 734.4 & 158.1 & 26 & 30 \\
\hline & & & 576.4 & & 20 \\
\hline \multirow[t]{2}{*}{ Clarithromycin } & 3.5 & 748.5 & 158.1 & 30 & 30 \\
\hline & & & 82.9 & & 46 \\
\hline \multirow[t]{2}{*}{ Cephalexin } & 3.43 & 348.0 & 158.0 & 14 & 8 \\
\hline & & & 174.0 & & 14 \\
\hline
\end{tabular}


at 6,12 , and $18 \mathrm{~h}$. Cephalexin, cefotaxime, clarithromycin, and doxycycline were selected for this purpose as they were more extensively degraded than the other drugs tested. The amount of antimicrobial degraded during each $6 \mathrm{~h}$ step was calculated for each antimicrobial and each well. The computed lost amounts were added in $5 \mu \mathrm{L}$ to each $200 \mu \mathrm{L}$ well, designated "fortified well," at each corresponding time in an attempt to compensate for this degradation. Escherichia coli ATCC 25922 and Staphylococcus aureus HG001 strains were used as test organisms. The MBC (Marchbanks et al., 1987; Clinical and Laboratory Standards Institute, 1999) for each organism was assessed by counting the viable bacteria in the broth from all wells that inhibited visible growth at $24 \mathrm{~h}$. Bacteria were counted in triplicate on tryptic soy agar supplemented with magnesium sulfate and activated charcoal to prevent antibiotic carry-over effects. Colonies were counted after overnight incubation at $37^{\circ} \mathrm{C}$. The lowest concentration of the antimicrobial that killed $\geq 99.9 \%$ of the initial inoculum was defined as the MBC end point. MBC and MIC were determined in triplicate at $24 \mathrm{~h}$ and the values for the control and fortified wells were compared by Wilcoxon test, with statistical significance set at $p \leq 0.05$.

\section{RESULTS}

\section{Analytical Results}

The UPLC conditions were optimized to ensure the rapid elution of all 7 antimicrobials with reliable retention times and MS ionizations to avoid storage in the autosampler. The elution was performed on a C18 column within $5 \mathrm{~min}$ and detection was by MRM in positive electrospray ionization (ESI+). All 7 antimicrobials gave the protonated parent $[\mathrm{M}+\mathrm{H}]^{+}$with two MRM transitions. The MRM transition with the highest intensity was selected for quantification and the second MRM transition was used for antimicrobial confirmation. These two MRM transitions, the precise time of retention, in addition to the absence of interference in the MHB blank samples corroborated the high selectivity of the method.

Calibration ranges were chosen to include the MIC most frequently reported by EUCAST for Staphylococcus aureus strains. They also included the reported ECOFF values for $S$. aureus strains, except for amoxicillin for which the E. coli ECOFF value was chosen as no data for $S$. aureus were available (EUCAST, 2016). The LOQ was established in order to be able to quantify at least $90 \%$ of antimicrobial degradation (except for doxycycline). The validation results are reported in Table 2. The accuracy for all 7 antimicrobials ranged from 92 to $111 \%$ with a precision of less than $18 \%$. LOQ were validated at $0.01 \mu \mathrm{g} / \mathrm{mL}$ for cefotaxime, ciprofloxacin and the two macrolides clarithromycin and erythromycin, at $0.05 \mu \mathrm{g} / \mathrm{mL}$ for amoxicillin and doxycycline and at $0.1 \mu \mathrm{g} / \mathrm{mL}$ for cephalexin. The accuracy at the LOQ ranged from 80 to $120 \%$ with a precision lower than $18 \%$.

\section{Degradation of Different Antibiotics during the Broth Microdilution Test}

The concentrations tested were 2,4 , and $8 \mu \mathrm{g} / \mathrm{mL}$ for amoxicillin (Figure S1) and cephalexin, 1, 2, and $4 \mu \mathrm{g} / \mathrm{mL}$ for cefotaxime, $0.25,0.5$, and $1 \mu \mathrm{g} / \mathrm{mL}$ for ciprofloxacin, 0.125, 0.25, and
$0.5 \mu \mathrm{g} / \mathrm{mL}$ for erythromycin and clarithromycin and 0.0625 , 0.125 , and 0.25 for doxycycline. The degradation half-lives and percentages of degradation of the seven antimicrobials, measured after incubation for $24 \mathrm{~h}$ using the standard broth microdilution test conditions, are given in Table 3. All these drugs were unstable (degradation $>20 \%$ ) at the above concentrations and conditions. Some drugs, such as clarithromycin and doxycycline hyclate, were highly degraded (more than 60\%). Others, such as amoxicillin, cephalexin monohydrate, cefotaxime sodium salt and erythromycin hydrate were moderately degraded (from 40 to $60 \%$ ) while ciprofloxacin showed the least degradation (less than $40 \%)$.

\section{Consequences of Antibiotic Degradation on MIC and MBC Determinations}

The MBC and MIC values for each test organism and each drug are given in Table 4. The MIC and MBC values obtained in the fortified wells were either equal to or lower than those obtained under standard conditions (no compensation for drug lost). The AST values were significantly different for one drug out of 4 for each investigated organism. However, the differences, unrelated to the presence of microorganisms, never exceeded more than one dilution.

\section{DISCUSSION}

The stability or instability of the different antimicrobials used in our study has already been reported in various liquid and solid media (Hwang et al., 1986; Marchbanks et al., 1987; Paesen et al., 1994; Erah et al., 1997). Erah et al. showed that amoxicillin and clarithromycin are stable at $\mathrm{pH} 7$ in aqueous solution with a degradation half-life of respectively $153.1 \mathrm{~h}$ and undetectable degradation (Erah et al., 1997). However, the stability of a given drug in one set of conditions cannot be univocally extrapolated to other conditions. Indeed, in our experiment, the degradation half-lives of amoxicillin and clarithromycin in $\mathrm{MHB}$ at $37^{\circ} \mathrm{C}$ were 27.4 and $14.2 \mathrm{~h}$ respectively. Cefotaxime stability in solution at $24 \mathrm{~h}$ is reported to be $92 \%$ at $25^{\circ} \mathrm{C}$ and $72 \%$ at $45^{\circ} \mathrm{C}$ (Behin et al., 2012). The extent of degradation in $\mathrm{MHB}$ is greater as $44.7 \%$ of the drug was degraded at $24 \mathrm{~h}$ and $37^{\circ} \mathrm{C}$. While it is clear that light, temperature, and $\mathrm{pH}$ are important factors to consider, the test medium can also influence stability of a compound (Paesen et al., 1994). Cielecka-Piontek et al. did study the effect of different injection media on stability of meropenem and clavulanate potassium in order to allow clinical administration of both compound together while maximizing stability and compatibility (Cielecka-Piontek et al., 2015). There were clear differences between media, indicating the use of one media more than others in a clinical setting even if all of them where aqueous. Differences between media can be explained by their nature (aqueous or lipophilic) or differences in the catalytic effects of degradation products(Cielecka-Piontek et al., 2015). Wick stated in 1964 that every new antibiotic should be subjected to a complete study of stability under test conditions (Wick, 1964). 
TABLE 2 | Validation results for seven different antimicrobials in MHB.

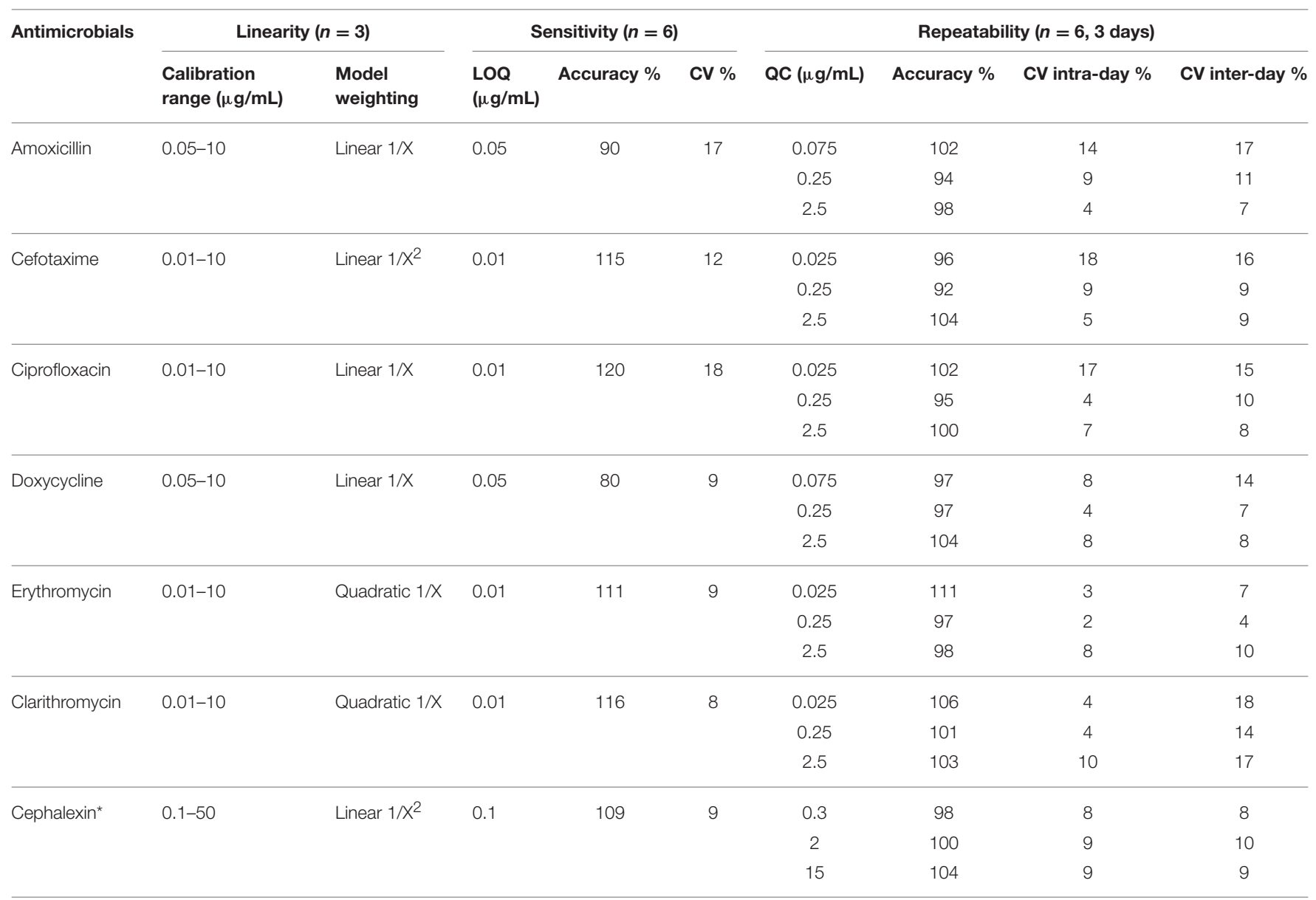

${ }^{\star}$ Cephalexin was assayed with a previous existing method and repeatability was evaluated on 2 days.

TABLE 3 | Half-lives and percentages of degradation after $24 \mathrm{~h}$ for different antimicrobials under the same conditions as in the MIC determination, apart from the absence of bacteria.

\begin{tabular}{lcc}
\hline Antimicrobial & $\begin{array}{c}\text { Degradation } \\
\text { half-life } \mathbf{( h ) ^ { * }}\end{array}$ & $\begin{array}{c}\text { Percentage of degradation } \\
\text { after 24 } \mathbf{~ ( \% )}\end{array}$ \\
\hline Amoxicillin & 27.4 & 45.5 \\
Cefotaxime & 28.1 & 44.7 \\
Ciprofloxacin & 42.0 & 32.7 \\
Doxycycline & 16.9 & 62.6 \\
Erythromycin & 31.5 & 41.0 \\
Clarithromycin & 14.2 & 69.0 \\
Cephalexin & 20.2 & 56.1 \\
\hline
\end{tabular}

*Half-life was estimated using a monoexponential model to describe the observed decay. The means of 3 experiments are reported.

Previous studies have shown that antimicrobials can be degraded during the storage of MIC trays depending on the selected drug and storage time and that a low temperature $\left(-70^{\circ} \mathrm{C}\right)$ is usually best for storage (Barry et al., 1976; Hwang et al., 1986; Marshall et al., 2000).
Problems with imipenem stability have been reported during storage of MIC trays (Nickolai et al., 1985), but it did not occured with meropenem in the same conditions (Dowzicky et al., 1994) despite also being a carbapenem, attesting the need to study each compound in a given condition. It also highlighted that every precaution should be taken to minimize the loss of moisture from the frozen trays (Barry et al., 1976). White et al. demonstrated that antimicrobial degradation during storage can also result in a decline of susceptibility as the MIC values increase with degradation and storage time (White et al., 1991). Our results confirmed that antimicrobial degradation during incubation can lead to a significant increase in the observed MIC or MBC values and hence to a bias when reporting AST results.

AST plays a major role in predicting the efficacy of an antibiotic against clinical pathogens and thus in its subsequent selection (Craig, 1998). As our study with the standard microdilution method showed that some widely-used antibiotics were degraded during incubation, we explored the possible consequences of a biased MIC estimate on AST interpretation. AST results are assigned to one of three categories (susceptible, 
TABLE 4 | MIC and MBC data for 4 unstable antimicrobials against Escherichia coli and Staphylococcus aureus at $24 \mathrm{~h}$ with the standard microdilution method or with ad-hoc addition of antimicrobial every $6 \mathrm{~h}$ to compensate for degradation.

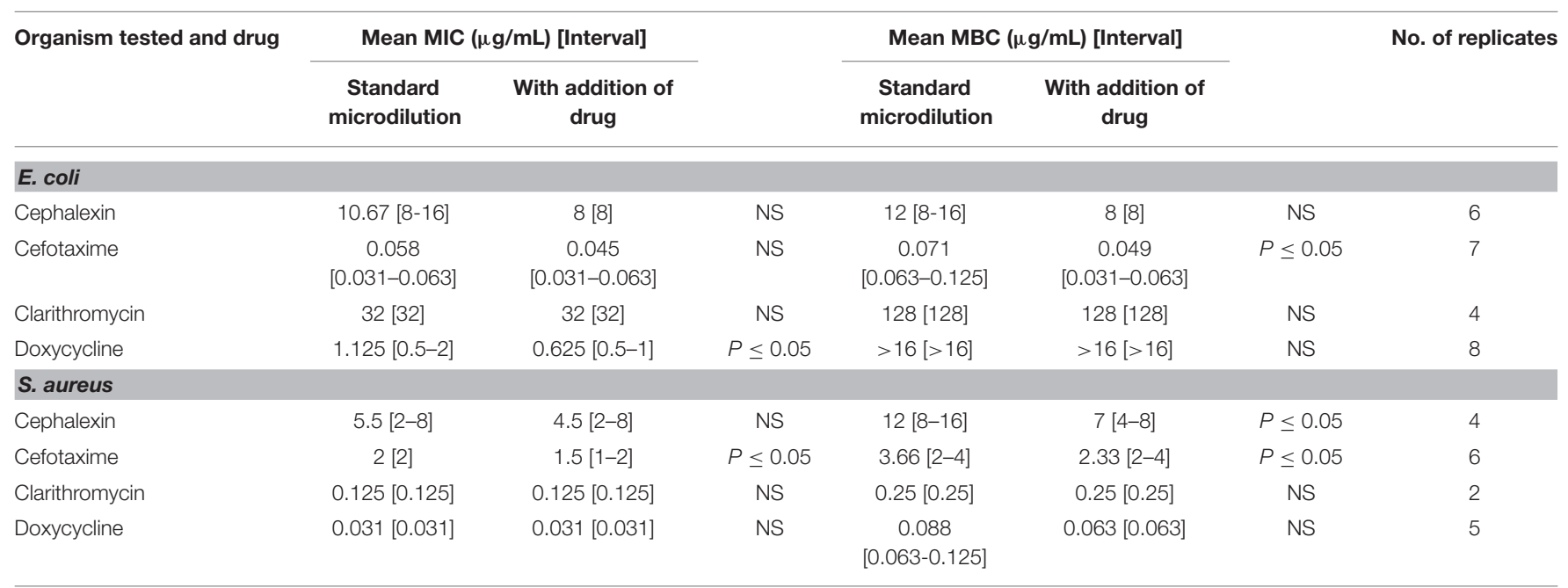

Groups significantly different $(p \leq 0.05)$ or not (NS).

intermediate, or resistant) based on two critical lower and upper MIC breakpoints. The susceptible and resistant classes are commonly separated by an intermediate class corresponding to just one twofold dilution (Annis and Craig, 2005). Given the narrowness of this intermediate range, such a factor of variability can seriously impact the correct classification of a pathogen if its MIC is near the breakpoints (Craig, 2000). $\mathrm{PK} / \mathrm{PD}$ indices such as AUC/MIC are used to establish optimal dosing regimens and are calculated from the MIC reported for the pathogen (Craig, 1998). The impact of an incorrect MIC on the dosage regimen calculation can thus be very real.

Nevertheless, it has to be kept in mind that, in our study, the differences in MIC or MBC values due to antimicrobial degradation was never higher than one twofold dilution even if $69 \%$ degradation of clarithromycin could have suggested a higher impact on MIC and MBC values. This small effect on AST values could be related to the short bacterial generation times, which are 25 and $29 \mathrm{~min}$, respectively, for our strains of E. coli and $S$. aureus in MHB (personal data), compared to antimicrobial degradation half-lives that are quite long in comparison to those doubling times in our study. Furthermore, a MIC value is not a simple concentration, unlike the value measured by HPLC, and actually represents the overall effect obtained over the entire incubation time. Repeated measurements of MIC using the same pathogen/drug combination commonly show a 3-fold dilution range, meaning that the standard microdilution method has an accuracy of not less than one dilution interval and that a one dilution difference can be considered as acceptable (Wexler et al., 1990; Annis and Craig, 2005).

In some specific situations requiring accurate $\mathrm{MIC}$ and MBC values, or when the bacterial doubling time is higher than the drug half-life, as with Mycobacterium tuberculosis
(Srivastava et al., 2016), accurate data may be obtained by controlling the antimicrobial concentration to balance antibiotic degradation. Srivastava et al. recently described a method for measuring the MIC of ertapenem against $M$. tuberculosis (Srivastava et al., 2016). They used tubes with bacteria and different concentrations of antimicrobial and added a daily supplement to compensate for ertapenem degradation. The resulting MIC was far lower than the MIC obtained with noncompensated tubes ( 0.6 and $\mathrm{mg} / \mathrm{L}$ respectively). Another option is to plot bactericidal time-kill curves by using an initial bacterial inoculum of $510^{5} \mathrm{UFC} / \mathrm{mL}$ and varying the concentrations of antimicrobial. The interaction between the antimicrobial and the bacteria can be modeled (Nielsen and Friberg, 2013) and drug degradation could be considered in the modeling process (Nielsen and Friberg, 2013). The MIC obtained with this method would correspond to the concentration of antimicrobial for which a final inoculum of $510^{5} \mathrm{UFC} / \mathrm{mL}$ is obtained at 20 or $24 \mathrm{~h}$. Such methods are interesting for specific cases but are time-consuming and cannot be applied to standard MIC determinations. In this study, we only considered the possible action of an abiotic MHB culture medium on antimicrobial degradation, as measured by UPLC, using the strict conditions applied during microdilution testing. Antibiotic degradation during such testing can also be due to metabolism, as with serum esterases and cefotaxime, for example, (Marchbanks et al., 1987), or to the effect of microorganisms in the system (Wick, 1964). Another potential source of variation for MIC determination can be the cation concentration variability in Mueller-Hinton broth (Clinical and Laboratory Standards Institute, 1999). This problem was overcome in our study by using the same cation-adjusted Mueller-Hinton broth for all experiments.

On the basis of our results, we conclude that the microdilution method remains suitable for testing the antimicrobial 
susceptibility of most common pathogens, despite the concomitant degradation of some antimicrobials.

\section{AUTHOR CONTRIBUTIONS}

EL, PT, SB, AF, and AB conceived and designed the experiments. $\mathrm{EL}$ and $\mathrm{ML}$ performed the experiments. $\mathrm{EL}, \mathrm{ML}, \mathrm{AF}$, and $\mathrm{AB}$ analyzed the data. EL, ML, PT, SB, AF, and AB wrote the paper.

\section{FUNDING}

The experimental assays were supported by CEVA-Sogeval (France). The funders had no role in study design, data collection

\section{REFERENCES}

Almeida, A. M., Castel-Branco, M. M., and Falcão, A. C. (2002). Linear regression for calibration lines revisited: weighting schemes for bioanalytical methods. J. Chromatogr B Anal. Technol. Biomed. Life Sci. 774, 215-222. doi: 10.1016/S1570-0232(02)00244-1

Annis, D. H., and Craig, B. A. (2005). The effect of interlaboratory variability on antimicrobial susceptibility determination. Diagn. Microbiol. Infect. Dis. 53, 61-64. doi: 10.1016/j.diagmicrobio.2005.03.012

Barry, A. L., Effinger, L. J., and Badal, R. E. (1976). Short-term storage of six penicillins and cephalothin in microdilution trays for antimicrobial susceptibility tests. Antimicrob. Agents Chemother. 10, 83-88. doi: 10.1128/AAC.10.1.83

Behin, S., Punitha, I. S. R., and Krishnan, S. (2012). Physical and chemical stability studies on cefotaxime and its dosage forms by stability indicating HPTLC method. Int. J. Pharma. Chem. Biol. Sci. 2, 517-523. Available online at: http:// www.ijpcbs.com/files/volume2-4-2012/15.pdf

Cielecka-Piontek, J., Szymanowska-Powalowska, D., Paczkowska, M., Lysakowski, P., Zalewski, P., and Garbacki, P. (2015). Stability, compatibility and microbiological activity studies of meropenem-clavulanate potassium. J. Antibiot. 68, 35-39. doi: 10.1038/ja.2014.92

Clinical and Laboratory Standards Institute (1999). M26-A Methods for Determining Bactericidal Activity of Antimicrobial Agents; Approved Guideline. Wayne, PA.

Clinical and Laboratory Standards Institute (2012). Methods for Dilution Antimicrobial Susceptibility Tests for Bacteria That Grow Aerobically; Approved Standard-Ninth Edition. Wayne, PA: Clinical and Laboratory Standards Institute, 68.

Craig, B. A. (2000). Modeling approach to diameter breakpoint determination. Diagn. Microbiol. Infect. Dis. 36, 193-202. doi: 10.1016/S0732-8893(99)00130-3

Craig, W. A. (1998). Pharmacokinetic/pharmacodynamic parameters: rationale for antibacterial dosing of mice and men. Clin. Infect. Dis. 26, 1-10. doi: $10.1086 / 516284$

Dowzicky, M. J., Nadler, H. L., and Sheikh, W. (1994). Comparison of sensititre broth microdilution and agar dilution susceptibility testing techniques for meropenem to determine accuracy, reproducibility, and predictive values. J. Clin. Microbiol. 32, 2204-2207.

Erah, P. O., Goddard, A. F., Barrett, D. A., Shaw, P. N., and Spiller, R. C. (1997). The stability of amoxycillin, clarithromycin and metronidazole in gastric juice: relevance to the treatment of Helicobacter pylori infection. J. Antimicrob. Chemother. 39, 5-12. doi: 10.1093/jac/39.1.5

EUCAST (2016). Antimicrobial Wild Type Distributions of Microorganisms: Staphylococcus aureus [cited 2016 09/21/2016]. Available online at: http:// mic.eucast.org/Eucast2/SearchController/search.jsp?action=performSearch\& BeginIndex $=0 \&$ Micdif $=$ mic $\&$ NumberIndex $=50 \&$ Antib $=-1 \&$ Specium $=14$

Hwang, J. M., Piccinini, T. E., Lammel, C. J., Hadley, W. K., and Brooks, G. F. (1986). Effect of storage temperature and $\mathrm{pH}$ on the stability of antimicrobial agents in MIC trays. J. Clin. Microbiol. 23, 959-961.

ICH (1994). International Conference on Harmonisation of Technical Requirements for Registration of Pharmaceuticals for Human Use. ICH Harmonised Tripartite or interpretation, or in the decision to submit the work for publication.

\section{ACKNOWLEDGMENTS}

We thank Nathalie Arpaillange for technical assistance in bacteriology.

\section{SUPPLEMENTARY MATERIAL}

The Supplementary Material for this article can be found online at: http://journal.frontiersin.org/article/10.3389/fmicb. 2016.02051/full\#supplementary-material
Guideline Validation of Analytical Procedures: Text and Methodology Q2(R1) [cited 2016 09/21/2016]. Step 4 Version:Available online at: http://www.ich.org/ fileadmin/Public_Web_Site/ICH_Products/Guidelines/Quality/Q2_R1/Step4/ Q2_R1_Guideline.pdf

Marchbanks, C. R., Yost, R. L., and White, R. L. (1987). Cefotaxime stability during in vitro microbiological testing. Antimicrob. Agents Chemother. 31, 1375-1378. doi: 10.1128/AAC.31.9.1375

Marshall, S. A., Kugler, K. C., and Jones, R. N. (2000). Evaluation of quinupristin/dalfopristin (Synercid) and RPR 106972 stability in susceptibility testing media. Int. J. Antimicrob. Agents 15, 291-297. doi: 10.1016/S0924-8579 (00)00180-1

Nickolai, D. J., Lammel, C. J., Byford, B. A., Morris, J. H., Kaplan, E. B., Hadley, W. K., et al. (1985). Effects of storage temperature and $\mathrm{pH}$ on the stability of eleven beta-lactam antibiotics in MIC trays. J. Clin. Microbiol. 21, 366-370.

Nielsen, E. I., and Friberg, L. E. (2013). Pharmacokinetic-pharmacodynamic modeling of antibacterial drugs. Pharmacol. Rev. 65, 1053-1090. doi: 10.1124/ pr.111.005769

Paesen, J., Quintens, I., Thoithi, G., Roets, E., Reybrouck, G., and Hoogmartens, J. (1994). Quantitative analysis of quaternary ammonium antiseptics using thin-layer densitometry. J. Chromatogr. A. 677, 377-384. doi: 10.1016/0021-9673(94)80165-7

Srivastava, S., van Rijn, S. P., Wessels, A. M. A., Alffenaar, J. W. C., and Gumbo, T. (2016). Susceptibility testing of antibiotics that degrade faster than the doubling time of slow-growing mycobacteria: ertapenem sterilizing effect versus Mycobacterium tuberculosis. Antimicrob. Agents Chemother. 60, 3193-3195. doi: 10.1128/AAC.02 924-15

Wexler, H. M., Lavin, P. T., Molitoris, E., and Finegold, S. M. (1990). Statistical analysis of the effects of trial, reader, and replicates on MIC determination for cefoxitin. Antimicrob. Agents Chemother. 34, 2246-2249. doi: 10.1128/AAC.34.11.2246

White, R. L., Kays, M. B., Friedrich, L. V., Brown, E. W., and Koonce, J. R. (1991). Pseudoresistance of Pseudomonas aeruginosa resulting from degradation of imipenem in an automated susceptibility testing system with predried panels. J. Clin. Microbiol. 29, 398-400.

Wick, W. E. (1964). Influence of antibiotic stability on the results of in vitro testing procedures. J. Bacteriol. 87, 1162-1170.

Conflict of Interest Statement: The authors declare that the research was conducted in the absence of any commercial or financial relationships that could be construed as a potential conflict of interest.

Copyright (c) 2016 Lallemand, Lacroix, Toutain, Boullier, Ferran and BousquetMelou. This is an open-access article distributed under the terms of the Creative Commons Attribution License (CC BY). The use, distribution or reproduction in other forums is permitted, provided the original author(s) or licensor are credited and that the original publication in this journal is cited, in accordance with accepted academic practice. No use, distribution or reproduction is permitted which does not comply with these terms. 